\title{
The future of quantitative pupillometry in health and disease
}

\author{
Vanessa Troiani ${ }^{1,2,3,4}$
}

Received: 6 December 2019 / Accepted: 9 December 2019 / Published online: 3 January 2020

(c) Springer-Verlag GmbH Germany, part of Springer Nature 2020

The amount of light that reaches the retina is regulated through the synergistic constriction and dilation of the pupil in response to the amount of ambient light in a given environment. The pupillary light reflex (PLR) describes the rapid, immediate constriction of the pupil that is evoked by flashing a short-duration light stimulus directly into a person's eye, followed by rapid dilation following stimulus offset. Although the PLR can be observed by flashing a simple penlight, quantitative pupillometry systems allow for a more objective measurement of the response as well as better stimulus control. Further, quantitative pupillometry systems compute several other parameters that may reflect individual variation in the PLR, including latency and velocity of the response.

The PLR is controlled by relatively simple sensory circuits, with many aspects of the constriction portion of the PLR under direct control of the parasympathetic nervous system and highly responsive to acetylcholine. In contrast, dilation of the pupil following the removal of a light stimulus is modulated by central and peripheral sympathetic inputs that are largely norepinephrine-dependent. Given these complementary innervations, the measurement of dynamic aspects of the PLR can serve as a noninvasive proxy for parasympathetic and sympathetic nervous system balance. Quantitative PLR methods may also represent an avenue through which to capture the dysregulation of these systems underlying several clinical disorders.

In this issue of Clinical Autonomic Research, Winston and colleagues assess the PLR in over 300 healthy pediatric

Vanessa Troiani

vtroiani@geisinger.edu

1 Autism and Developmental Medicine Institute, Geisinger, 120 Hamm Drive, Suite 2A, Lewisburg, PA 17837, USA

2 Neuroscience Institute, Geisinger, Danville, PA, USA

3 Department of Imaging Science and Innovation, Geisinger, Danville, PA, USA

4 Department of Basic Sciences, Geisinger Commonwealth School of Medicine, Scranton, PA, USA participants, aged 2-21 years [1]. This study represents the largest prospective analysis using quantitative pupillometry in a healthy pediatric cohort. With the use of a handheld pupillometer, monocular recordings were obtained from each eye, and the subcomponents of the PLR (including size, latency, and velocity metrics) were analyzed. Interestingly, the authors found that the PLR did not change with development in a simple linear manner. Rather, specific dynamic aspects of the PLR were best modeled by different polynomial functions over time, with the percentage of constriction and dilation velocity following a linear trend, initial pupil diameter following a quadratic trend, and constriction velocity maturing cubically. These results highlight the importance of assessing more complex relationships between physiological responses and age.

In addition to confirming that there are age-related changes in the PLR during child development, the data generated by Winston and colleagues can serve as normative benchmarks for use in future research. Given the differences in PLR variables between those in different developmental stages, age-specific normative values may be particularly important when the PLR is obtained for clinical purposes. Although the clinical utility of the PLR is continually expanding, it is already in use in a variety of clinical settings to assess brain function at the individual level. Within inpatient settings, pupillometers can be useful for determining whether a patient with a traumatic brain injury is stable or may require additional intervention. In emergency settings, the PLR can indicate the use of certain medications (barbiturates and opioids) or suggest optic nerve injury and/ or brain stem tumors.

More recently, the potential utility of the PLR and other pupil measurements has been found to have neurodiagnostic value as a biomarker for specific disorders or conditions. For example, children with autism spectrum disorder may have an atypical PLR [2] and atypical response to light and dark stimuli [3], with atypical pupil responses present even before diagnosis [4]. One particularly important and intriguing aspect in using pupillary responses as a metric in pediatric populations is that this is a measure that is objective 
and does not rely on language skills, which may not be fully developed in young children or those with neurodevelopmental disabilities. Given the potential that PLR measurement offers in pediatric populations, the study by Winston and colleagues proves timely in generating normative data that spans infancy through early adulthood.

Another area that may benefit from using pupillary response metrics is the evaluation of acute concussion in both children and adults [5]. Some newer technologies allow for an individual to track multiple pupillary measurements on their own patient identification card or chip. This would allow student athletes to be assessed pre-season and would then allow for individual assessment in the case of a potential concussion. Consistent with this idea, a recent study found significant differences in PLR velocity following high-acceleration head impact in high school football athletes who were otherwise clinically asymptomatic [6]. It is important to note that there are additional clinical applications for pupillometry that extend beyond pediatric populations. Significant differences have been observed in various PLR parameters between healthy individuals and those with Alzheimer's disease or Parkinson's disease [7, 8]. PLR measurements may also offer an objective tool for measuring impairment due to illicit drug or alcohol use or in the context of a treatment response [9].

Although the normative PLR values published by Winston and colleagues are a great first step in furthering PLR research in pediatric populations, the data were collected cross-sectionally. It will be important for future work to assess longitudinal changes in the pupillary response within the same individuals over the course of development, as well as to establish test-retest reliability in both pediatric and adult populations. The field is only beginning to understand the value of the PLR as a noninvasive, quantitative indicator of a wide-range of acute and chronic conditions.

Funding None.

\section{Compliance with ethical standards}

Conflict of interest The author declares that they have no conflict of interest.

\section{References}

1. Winston M, Zhou A, Rand CM, Dunne EC, Warner JJ, Volpe LJ, Pigneri BA, Simon D, Bielawiec T, Gordon SC, Vitez SF, Charnay A, Joza S, Kelly K, Panicker C, Rizvydeen S, Niewijk G, Coleman C, Scher BJ, Reed DW, Hockney SM, Buniao G, Stewart T, Trojanowski L, Brogadir C, Price M, Kenny AS, Bradley A, Volpe NJ, Weese-Mayer DE (2019) Pupillometry measures of autonomic nervous system regulation with advancing age in a healthy pediatric cohort. Clin Auton Res 2019:1. https://doi. org/10.1007/s10286-019-00639-3 (In Press)

2. Fan X, Miles JH, Takahashi N, Yao G (2009) Abnormal transient pupillary light reflex in individuals with autism spectrum disorders. J Autism Dev Disord 39(11):1499-1508

3. DiCriscio AS, Troiani V (2017) Pupil adaptation corresponds to quantitative measures of autism traits in children. Sci Rep $7(1): 6476$

4. Nyström P, Gliga T, Jobs EN, Gredebäck G, Charman T, Johnson MH, Falck-Ytter T et al (2018) Enhanced pupillary light reflex in infancy is associated with autism diagnosis in toddlerhood. Nature Commun 9(1):1678

5. Capó-Aponte JE, Beltran TA, Walsh DV, Cole WR, Dumayas JY (2018) Validation of visual objective biomarkers for acute concussion. Mil Med 183(1):9-17

6. Joseph JR, Swallow JS, Willsey K, Almeida AA, Lorincz MT, Fraumann RK, Oppenlander ME, Szerlip NJ, Broglio SP (2019) Pupillary changes after clinically asymptomatic high-acceleration head impacts in high school football athletes. J Neurosurg 26:suppl_1-6. https://doi.org/10.3171/2019.7.JNS191272

7. Giza E, Fotiou D, Bostantjopoulou S, Katsarou Z, Karlovasitou A (2011) Pupil light reflex in Parkinson's disease: evaluation with pupillometry. Int J Neurosci 121(1):37-43

8. Fotiou DF, Brozou CG, Haidich AB, Tsiptsios D, Nakou M, Kabitsi A, Fotiou F et al (2007) Pupil reaction to light in Alzheimer's disease: evaluation of pupil size changes and mobility. Aging Clin Exp Res 19(5):364-371

9. Jochum T, Hoyme J, Schulz S, Weißenfels M, Voss A, Bär KJ (2016) Diverse autonomic regulation of pupillary function and the cardiovascular system during alcohol withdrawal. Drug Alcohol Depend 159:142-151 